\title{
Genome editing of immune cells using CRISPR/Cas9
}

\author{
Segi Kim, Cedric Hupperetz, Seongjoon Lim \& Chan Hyuk Kim* \\ Department of Biological Sciences, Korea Advanced Institute of Science and Technology (KAIST), Daejeon 34141, Korea
}

\begin{abstract}
The ability to read, write, and edit genomic information in living organisms can have a profound impact on research, health, economic, and environmental issues. The CRISPR/Cas system, recently discovered as an adaptive immune system in prokaryotes, has revolutionized the ease and throughput of genome editing in mammalian cells and has proved itself indispensable to the engineering of immune cells and identification of novel immune mechanisms. In this review, we summarize the CRISPR/ Cas9 system and the history of its discovery and optimization. We then focus on engineering $T$ cells and other types of immune cells, with emphasis on therapeutic applications. Last, we describe the different modifications of Cas9 and their recent applications in the genome-wide screening of immune cells. [BMB Reports 2021; 54(1): 59-69]
\end{abstract}

\section{INTRODUCTION}

Recent advances in technology that enables us to read, write, and edit genetic information have revolutionized our understanding of biology. For example, the introduction of next-generation sequencing (NGS) beyond Sanger sequencing in the mid-2000s has transformed the field of genomics because we can retrieve vast amounts of cellular genomic, epigenomic, and transcriptomic information at high speeds and low costs (1). In addition, decades of research efforts on synthetic genomes have enabled the complete synthesis of bacterial genomes (2). For genome editing, engineered nucleases, such as zinc finger nucleases (ZFN) and transcription activator-like effector nucleases (TALEN), have laid the foundation for executing site-specific genetic alterations (3). In contrast to ZFNs and TALENs, which require complicated protein engineering for customizing DNAbinding domains, the CRISPR/Cas system relies on short guide RNAs (gRNA) to target any desired location in the genome (4). As such, this system has popularized genome editing for various

*Corresponding author. Tel: +82-42-350-2621; Fax: +82-42-350-2610; E-mail: Kimchanhyuk@kaist.ac.kr

https://doi.org/10.5483/BMBRep.2021.54.1.245

Received 21 October 2020, Revised 16 November 2020, Accepted 26 November 2020

Keywords: CRISPR/Cas9, Engineered T cells, Genome editing, Genome-wide screening, HSPC engineering biological studies, from basic research to therapeutic applications.

Further, the Cas nucleases have been modified to include fused adapter proteins, such as deaminases, transcriptional activators/repressors, and reverse transcriptase (5). With these adaptations, CRISPR/Cas systems have gained popularity in genomewide screening, wherein individual or epistatic factors involved in disease can quickly be discovered for future therapeutic strategies. In parallel, delivery methods have been optimized for more efficient gene editing, allowing for in vivo gene editing (6) against monogenic diseases and ex vivo production of engineered immune cells for treatment against common diseases like cancer or autoimmunity.

In this review, we discuss recent advances and potential strategies for engineering immune cells using CRISPR/Cas9 with a therapeutic emphasis. We review its brief history and functional mechanism and explore how this system has been applied for engineering $T$ and non- $T$ immune cells. Last, we review how CRISPR/Cas9-based screening has been used to find potential targets for immunotherapy and to uncover previously unknown immune pathways.

\section{GENOME EDITING WITH CRISPR/CAS9: HISTORY AND MECHANISM}

In 1987, Ishino et al. identified an unknown repeated sequence in Escherichia coli (7). Later, other groups independently reported similar repeated arrays in various species of bacteria and archaea, suggesting that they may play an important role. In 2002, the repeated sequence was named CRISPR, for clustered regularly interspaced short palindromic repeats, and the CRISPR-associated (Cas) gene cassette was identified near its locus (8). Subsequently in 2005, it was discovered that embedded within these CRISPR arrays were sequences originating from bacteriophages and conjugative plasmids, providing initial evidence of immune function (9). In 2007, it was experimentally proven that the CRISPR/Cas system provides protection against viruses in prokaryotes much as the RNA interference system does for eukaryotic cells (10).

The CRISPR/Cas system can be classified into Classes I and II. In the class I system, multiple Cas effector proteins mediate interference with invading genetic material, whereas in the class II system, a single Cas effector fulfills all effector functions (11). Of particular interest, the Streptococcus pyogenes Cas9 (SpCas9), which belongs to the Class II system, was the

ISSN: 1976-670X (electronic edition)

Copyright (c) 2021 by the The Korean Society for Biochemistry and Molecular Biology

(ㄷ) This is an open-access article distributed under the terms of the Creative Commons Attribution Non-Commercial License (http://creativecommons.org/licenses/by-nc/4.0) which permits unrestricted non-commercial use, distribution, and reproduction in any medium, provided the original work is properly cited. 
first to be investigated as a genome-editing tool in cells (12). Cas9 is a nuclease that forms a complex with either a CRISPR RNA (crRNA) and trans-activating crRNA (tracrRNA), or an engineered single-guide RNA (sgRNA) and uses them as a guide to target complementary genetic sequences for cleavage. The crRNA contains a short $\sim 20$-bp sequence that recognizes the target sequence. For Cas9-mediated cleavage, a short protospacer adjacent motif (PAM) sequence (5'-NGG-3') is required immediately downstream of the target sequence (13). Between the third and fourth nucleotides directly upstream of this PAM sequence, the phosphodiester bond is cleaved, resulting in a blunt-end double-stranded break (DSB) (12). For genome editing in eukaryotes, two major DSB repair mechanisms are exploited: nonhomologous end-joining (NHEJ) or homology-directed repair (HDR) (5). The NEHJ pathway is error-prone, with the ligation of two nascently cleaved DNA strands, accompanied by the addition or deletion of nucleotides, resulting in insertion-deletion (indel) mutations. The indel of an exon often induces frameshift mutation, resulting in knock out of gene. In contrast, HDR requires a homologous DNA sequence as a repair template and thus can do more precise editing compared to NHEJ (3). Of particular interest, HDR can be used to knock-in an exogenous donor sequence.

Further, the Cas9 protein has two independent nuclease domains: RuvC and $\mathrm{HNH}$. If either one is mutated, Cas9 exhibits only nickase activity (3). This mutant form can further be fused with deaminases for adenine/cytidine base editing and reverse transcriptase for prime editing (5). Similarly, mutation of both nuclease domains generates completely inactive Cas9 (dCas9) that can further be fused to other effector proteins, such as transcriptional activators or repressors, for programmable RNAguided epigenetic regulation (14).

Next, many CRISPR/Cas9 delivery methods have been developed. Cas9 can be delivered into cells by electroporation or chemical transfection of plasmid, RNA, or protein, or by using viral vectors, such as lentiviruses (LV) or adeno-associated viruses (AAV) (15). The gRNA can be delivered in a similar fashion: plasmid, viral vector, or as a ribonucleoprotein (RNP) complex with Cas9. Various combinations can be used, but for immunecell engineering, the most widely used method involves delivering RNP using electroporation, because of its instant activity and short half-life, which reduces off-target effects (16). Additionally, introduction of RNP can reduce cellular stress and toxicity better than can plasmid delivery (17) which can activate innate immune pathways within cells (18). For genomewide screening, the most common format involves the lentiviral delivery of sgRNA libraries into cells that stably express Cas9.

\section{CRISPR/CAS9-BASED GENOME EDITING FOR THERAPEUTIC T-CELL ENGINEERING}

Immunotherapy uses the immune system to fight multiple diseases, such as cancer, autoimmunity, and neurodegenerative diseases (19). For cancer, therapies that use T cells have been the most successful because of their target-specific and robust cytotoxic ability, rapid expansion, and long-term persistence in vivo. Examples include antibodies that engage $T$ cells, such as checkpoint inhibitors (20), bi-specific T-cell engagers (21), or ex vivo-expanded endogenous and genetically engineered $T$ cells (22). Genetic engineering of T cells mostly focuses on the transfer of additional receptors to redirect bulk $\mathrm{T}$ cells against specific cancer antigens. These can be recombinant tumor-reactive T-cell receptors (TCR) or chimeric antigen receptors (CAR), the latter of which is engineered by fusing an antigen-specific protein domain (usually scFv) with cytoplasmic signaling domains. CAR-T cells in particular do not rely on MHC-peptide recognition and instead can be redirected to any surface-bound tumor-associated antigen, and have been very successful in treating relapsed and refractory hematological malignancies (23). As such, two therapies, Kymriah (Tisagenlecleucel) and Yescarta (Axicabtagene ciloleucel), were approved in 2017 for the treatment of B-cell leukemias and lymphomas $(24,25)$. However, despite the initial success of engineered T-cell therapies, their efficacy, safety, and manufacturing need to be improved, all of which could potentially be addressed with CRISPR/Cas9-based genome-editing technology (Fig. 1).

\section{Gene knock-out with CRISPR/Cas9 in engineered T cells}

CRISPR/Cas9-based gene disruption has been used to enhance $\mathrm{T}$ cell activity, thereby increasing its efficacy. Among the targeted genes have been those involved in T-cell exhaustion, which is a dysfunctional state induced by repeated and chronic exposure to target antigens, as is often seen in many chronic viral infections and cancer (26). One major phenotypic change in exhausted $\mathrm{T}$ cells is the persistent expression of inhibitory checkpoint receptors, such as PD-1, CTLA-4, LAG-3, and TIGIT, which, upon ligation with their cognate ligands expressed on cancer cells or antigen-presenting cells, dampen the activation of T cells (27). Indeed, PD-1 blocking antibody therapy in the clinic has been effective against many cancers (20). PD-1 knockout by CRISPR/Cas9 has therefore been attempted and has shown increased activity of CAR-T cells in preclinical models and is also undergoing clinical evaluation (28-31). Likewise, its disruption is under investigation for TCR T therapy (32).

Next, transforming growth factor- $\beta$ (TGF- $\beta$ ) signaling in the tumor microenvironment suppresses the cytotoxic function of $\mathrm{CD} 8+\mathrm{T}$ cells and promotes the conversion of CD4 + T cells to the regulatory phenotype, negatively affecting anti-cancer immunity (33). Thus, blocking TGF- $\beta$ signaling might maintain the potency of transferred T cells within a suppressive tumor microenvironment. Indeed, the CRISPR/Cas9-mediated knock-out of the TFG- $\beta$ receptor 2 (TGFBR2) in CAR-T cells decreased induced regulatory $T$-cell $\left(T_{\text {reg }}\right)$ conversion and decreased the state of exhaustion during coincubation with cancer cells in the presence of TGF- $\beta 1$ (34). In addition, TGFBR2 knock-out in CAR-T cells showed improved tumor control in cell-line-derived and patient-derived xenograft mouse models with faster 


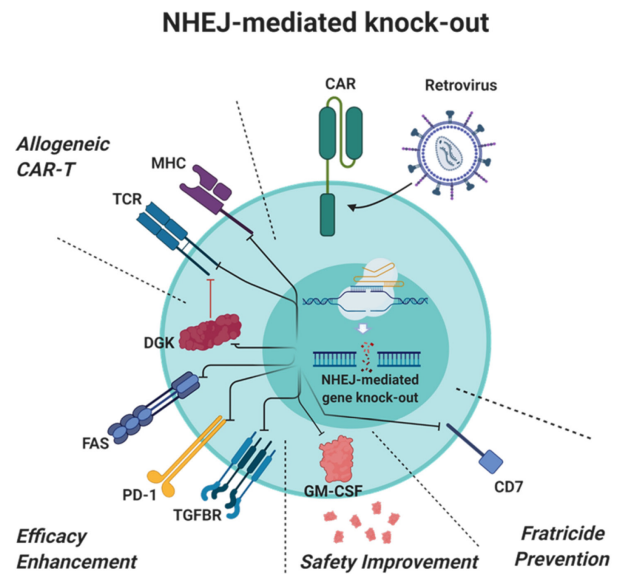

B

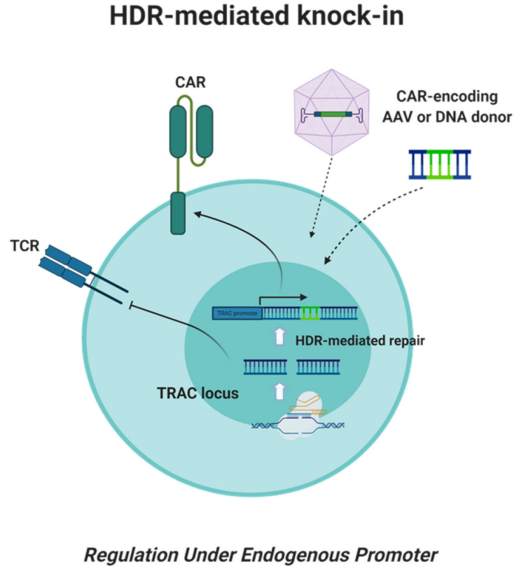

Fig. 1. CRISPR/Cas9 NHEJ- and HDR-mediated CAR-T modification. CRISPR/Cas9 NHEJ-mediated knock-out and HDR-mediated knock-in can be used for a variety of applications. For example in CAR-T cells, (A) NHEJ has been used to block alloreactivity to allogeneic receptors, to enhance the efficacy through deletion of immune checkpoint and death receptors, to prevent fratricide by deleting pan T-cell markers, and to increase safety by decreasing the potential for cytokine release syndrome. (B) Additionally, donor DNA deliver by AAV or dsDNA can be used for HDR-mediated knock-in of CARs into the TRAC locus, thereby creating CAR-T cells that have endogenous control over their CAR expression profiles. These donor templates contain the gene insert (green) which is flanked by two homologous arms (blue) that are complementary to the target locus.

tumor clearance, increased proportions of circulatory memory T-cell subsets, and increased persistence.

Activation-induced cell death (AICD) by death-receptor signaling is essential for central and peripheral tolerance by triggering T-cell apoptosis (35). The major death receptor involved in this process is Fas (CD95), which binds to Fas ligands (Fas-L). Repeated exposure of antigens to CAR-T cells activates Fas signaling, which induces apoptosis, and thereby hampers their anti-tumor activity. To address this, Ren et al. used CRISPR/Cas9 to deplete Fas, CD3, and $\beta-2$ microglobulin (B2M) to produce AICD-resistant universal CAR-T cells, which exhibited reduced apoptosis despite repeated antigen exposure and persisted in the peripheral blood of mice (36).

Diacylglycerol kinases (DGKs) are enzymes that catalyze the conversion of diacylglycerol (DAG) to phosphatidic acid (PA) (37). DAG is a central second messenger that is indispensable for $\mathrm{CD} 3$ signaling, by interacting with protein kinase $\mathrm{C}$ theta (PKC $\theta$ ) and Ras guanyl-releasing protein 1 (RasGRP1). Therefore, its conversion leads to decreased T-cell activation. DGK $\alpha$ and $\mathrm{DGK} \zeta$, two isoforms of DGK, are dominantly expressed in $\mathrm{T}$ cells, and their simultaneous deletion led to increased effector function and proliferation of CAR-T cells and increased resistance to immunosuppressive factors, such as TGF- $\beta$ and prostaglandin $\mathrm{E}_{2}$ (PGE-2) (38).

In addition to enhancing the efficacy of adoptive T-cell therapy, CRISPR/Cas9-mediated gene knock-out can be used to increase its safety. The rapid expansion of CAR-T cells in patients often leads to cytokine-release syndrome (CRS) and neurotoxicity due to the putative increase of inflammatory cytokines, such as IL-1 and IL-6 (39). In particular, IL-6 is mainly secreted by myeloid cells, such as monocytes. Rapidly expanding CAR-T cells secrete more granulocyte-macrophage colonystimulating factor (GM-CSF), which likely favors the activation of IL-6-secreting myeloid cells (40). Indeed, Sterner et al. demonstrated that neutralizing GM-CSF, using monoclonal antibodies, reduced CAR-T therapy-mediated CRS and neurotoxicity in a patient-derived xenograft model (41). Additionally, they applied a CRISPR/Cas9-based knock-out of GM-CSF in these CAR-T cells and found significantly decreased GM-CSF secretion upon activation without affecting their anti-tumor activity.

Next, CRISPR/Cas9 can be applied to the manufacturing of fratricide-resistant CAR-T cells, which is particularly important in the treatment of $\mathrm{T}$ leukemias and lymphomas. The most frequently targeted surface antigens for the treatment of these T-cell malignancies are the pan-T-cell markers CD3, CD5, and CD7 (42), which are also expressed on CAR-T cells, therefore making their manufacturing a challenge. Gomes-Silva et al. used CRISPR/Cas9 to delete CD7 prior to CAR delivery, rendering them fratricide-resistant, without altering their phenotype, effector function, or proliferative capacity. At the time of this review, these CAR-T cells are undergoing clinical evaluation (43).

Additionally, CRISPR/Cas9 can be used to create allogeneic off-the-shelf CAR-T products that have advantages over autologous products, potentially decreasing the manufacturing cost 
and narrowing the window between patient administration and treatment. The two main hurdles in allogeneic CAR-T therapy are graft-versus-host disease and graft rejection, both of which can be prevented by deletion of the TCR $\alpha$ and $\beta$ chains and B2M in donor T cells (44). Further, the disruption of endogenous TCR $\alpha$ and $\beta$ genes can be used to prevent mispairing with exogenous TCR, potentially enhancing the function and safety of TCR-T therapy (45).

\section{Targeted gene knock-in with CRISPR/Cas9 in engineered T cells}

In addition to disruption, CRISPR/Cas9 can be used to introduce genes of interest. Currently, the most widely used method in engineering $T$ cells for this purpose involves retroviral vectors such as $\gamma$-retrovirus (46) and lentivirus (47). Indeed, the two above-mentioned approved CAR-T products were produced using these vectors. These retroviral vectors can deliver transgenes with high efficiency while maintaining stable expression, but their random integration into the host genome comes with several challenges, such as the potential for insertional oncogenesis (48), and clonal expansion (49), and the variation in the integration copy number that results in heterogeneity of the engineered cell products (50), both of which can be overcome using site-specific integration with the CRISPR/Cas9 system.

Recently, Eyquem et al. integrated a CAR into the TCR $\alpha$ chain constant (TRAC) locus in T cells by introducing a DSB with mRNA-based CRISPR/Cas9 delivery and using an AAV6 vector for donor DNA delivery (51). Importantly, this method allowed for the disruption of the endogenous TCR $\alpha$ chain and for the expression of the CAR under the control of the endogenous TRAC promoter. This led to uniform CAR expression, decreased tonic signaling, optimal transcriptional regulation, and delayed T-cell differentiation and exhaustion, resulting in better tumor control in mouse xenograft models than from conventional retroviral CAR products. Additionally, the disruption of the TCR $\alpha$ chain has potential for off-the-shelf CAR T product manufacturing for the reasons explained above.

Although the delivery of donor DNA using non-integrating viral vectors such as AAV ensures high efficiency of knock-in, their production is a lengthy and complicated process that reduces their throughput (52). Additionally, the use of viral vectors can increase the manufacturing cost of engineered cell products and might have safety issues. To overcome this, nonviral donor DNA delivery may be preferred. Indeed, one study successfully used RNP and an electroporated DNA donor to insert a new TCR $\alpha / \beta$ chain (NY-ESO-1) into the TRAC locus (53). They optimized various parameters, including the stimulation conditions, the concentration of RNP and donor DNA template, and the electroporation parameters, thereby increasing the knock-in efficiency and reducing the toxicity in T cells. The knock-in efficiency of this method can be further improved by modifying the DNA donor template and stabilizing the RNP complex by adding anionic polymers (54). Next, the same group used a DNA-based donor strategy to compare the effects of 36 different genes (switch receptors, heterologous transcription factors, or metabolic regulators and receptors) inserted within the TRAC locus on the context-dependent fitness of human $T$ cells (55). As a result, they identified that the insertion of the TGFBR2-4-1BB chimeric receptor improved solidtumor clearance. Further optimization of DNA donor-based delivery methods will facilitate the basic research and clinical applications of CRISPR/Cas9-based knock-in technology in engineered T-cell therapy.

\section{CRISPR/CAS9-BASED GENOME EDITING IN OTHER IMMUNE-CELL TYPES}

CRISPR/Cas9 editing is not limited to the engineering of $\mathrm{T}$ cells. Indeed, many groups have used this system for disease modelling and therapeutic genome editing in non-T immune cells, such as hematopoietic stem and progenitor cells (HSPC) and $B$ cells (Fig. 2).

\section{Modeling hematological disorders}

Clonal hematopoiesis of intermediate potential (CHIP) is a premalignant state that involves the temporal acquisition of mutations in HSC that can lead to clonal expansion and an increased risk for hematological malignancies and cardiovascular diseases. To model CHIP, Tothova et al. used the CRISPR/Cas9 system in HSPC to introduce NHEJ-mediated disruption of DNMT3A, TET2, and ASXL1, and confirmed that these cells could be stably engrafted in immunodeficient mice, resulting in the highest clonal expansion over the course of 5 months (56). In addition, they used multiplexed editing in HSPCs to mutate 11 known leukemia drivers and found that mutation of RNX1, STAG2, SMC3, NF1, and DNM3TA led to the clonal expansion of immature myeloid cells in engrafted mice. Lastly, they performed a drug response test against HSPC with mutant TET2 or ASXL1 and found that TET2-mutated cells were more susceptible to azacytidine, a chemotherapy drug used to treat myelodysplastic syndrome.

In another study, Wagenblast et al. used CRISPR/Cas9 to assess the hypothesis that myeloproliferative disorders such as acute megakaryoblastic leukemia (AMKL) occurs in the developmental stage of HSPCs that can be derived from the unbalanced production of GATA1-Short and -Long isoforms. To model this, they used long-term HSC (LT-HSC), which is likely a source of clonal evolution in blood malignancies. They generated the GATA1-short isoform by DSB-mediated deletion of exon 2, and the GATA1-long isoform by introducing the additional start codon in exon 3 (57). With this model, they found that the two splice variants led to expanded LT-HSC clones with distinct differentiation and proliferation phenotypes.

\section{Therapeutic genome editing in HSPCs and B cells}

Genome editing has been used in HSPCs to correct $\beta$-hemoglobin disorders, which includes sickle-cell disease (SCD) and $\beta$-thalassemia, the former of which results from a missense 
A

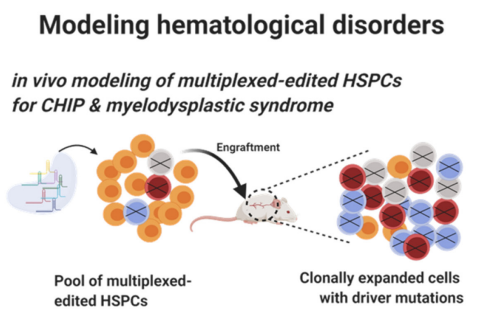

Clonal evaluation of two distinct GATA1 isoforms expressed in LT-HSCS for AMKL

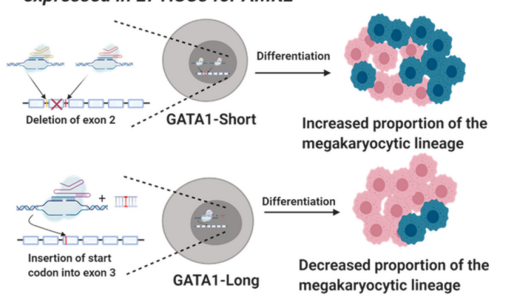

B

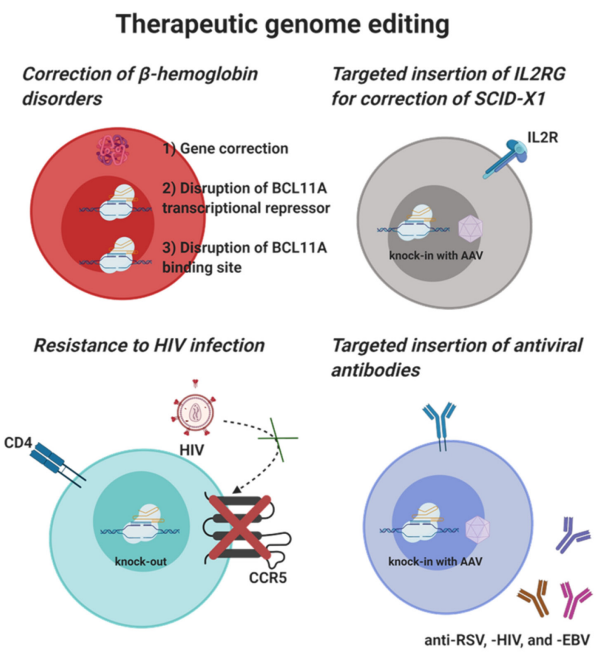

Fig. 2. Applications of CRISPR/Cas9 in HSPCs and B cells. CRISPR/Cas9 can be used to (A) model important pre-disease and disease states such as clonal hematopoiesis of indeterminate potential (CHIP), myelodysplastic syndrome, and acute megakaryoblastic leukemia (AMKL) and (B) to engineer therapeutic cells for the correction or disruption of disease-related genes alongside the introduction of disease-protective genes such as antiviral antibodies in B cells.

mutation in the $\beta$-hemoglobin gene, and the latter of which results from a nonsense or frameshift mutation (58). Using the CRISPR/Cas9 system, three different approaches have been used to treat these diseases: 1) the direct correction of mutant $\beta$-hemoglobin with HDR (59), 2) the disruption of the erythroid-specific enhancer of $\mathrm{BCL} 11 \mathrm{~A}$, a transcriptional repressor involved in the switching of fetal $\gamma$-hemoglobin to adult $\beta$-hemoglobin expression $(60,61)$, and 3$)$ the disruption of the BCL11A-repressor binding site in the $\gamma$-globin promoter $(62$, 63). In particular, approach 2) is being evaluated in clinical trials (NCT03745287, NCT03655678).

Next, severe combined immunodeficiency X1 (SCID-X1) is a disease caused by the loss-of-function mutation of the IL-2 receptor $\gamma$ chain (IL2RG), thereby impairing the development of $B, T$, and NK lymphocytes. Lentiviral delivery of wild-type IL2RG into HSCs and their subsequent adoptive transfer has been successful in recovering immune function in these patients. However, in previous clinical trials, the engineering of HSC using $\gamma$-retroviral vectors in a similar approach has led to T-cell leukemia in some patients due to insertional oncogenesis (64, 65). Although this has yet to be seen with the lentiviral system, Pavel-Dinu et al. (66) sought to site-specifically integrate wild-type IL2RG using AAV6 DNA donor delivery for HDRmediated repair, an approach that may circumvent safety concerns associated with retroviral vector-based delivery systems.

For infectious diseases, genome-editing approaches have been used to mitigate viral infections. For example, C-C chemokine receptor 5 (CCR5), an important co-receptor involved in human immunodeficiency virus (HIV) internalization into T cells and macrophages, is a promising target for therapy $(67,68)$. One study used CRISPR/Cas9 to delete CCR5 by using a dual sgRNA strategy in HSPCs and transplanted them into immunedeficient NPG mice (69). Following HIV-1 challenge, the group observed a significant decrease in the RNA of a CCR5-tropic HIV-1 strain in the blood plasma. Further, this group evaluated the clinical application of similarly prepared cells in a patient with HIV and acute lymphocytic leukemia and observed longterm albeit marginally efficient engraftment (NCT03164135) (70).

Further, like the introduction of exogenous TCRs or CARs in T-cell engineering, B cells can acquire exogenous antibody sequences with known antigen specificity. One group used CRISPR/Cas9 to introduce the sequence of a HIV-1 broadly neutralizing antibody that cannot be easily generated with conventional immunization (71). They showed that the adoptive transfer of these engineered B cells provided long-term protection in mice challenged with HIV-1. Similarly, another group knocked-in engineered neutralizing antibodies consisting of a single-chain Fab against respiratory syncytial virus (RSV), HIV, influenza virus, and Epstein-Barr virus in primary human and mouse B cells (72). The RSV-specific mouse B cells were tested in a mouse-infection model and showed neutralizing activity, providing further evidence that engineered B cells might be an alternative against viruses that do not yet have clinically available vaccines despite decades of efforts. 


\section{CRISPR/CAS9-BASED GENOME-WIDE SCREENING IN IMMUNE CELLS}

An important bottleneck in the research on immune mechanisms has been the low throughput of conventional genetic knock-out or knock-down strategies. The discovery and development of RNA interference (RNAi), particularly with shRNA technology that exploits endogenous RNAi machinery (73), has allowed for much higher throughput workflows, but these are still limited by the incomplete knock-down of targets or off-target interactions, which can make proper interpretation difficult (74). Furthermore, incomplete knock-down may make it difficult to properly identify lethal genes based on the viable sufficiency of incompletely inactivated gene expression (75). With the progressive development of CRISPR/Cas9 technology, many groups have developed platforms for genome-wide screening in non-immune and immune cells for robust and massively high-throughput experiments that allow for a rich understanding of cellular mechanisms (76).

Many different variations of the CRISPR/Cas9 screening system have been developed: classical gene knock-out using enzymatically active Cas9 (77), transcriptional activation (CRISPRa) with catalytically inactive Cas9 (dCas9) fused to transcriptional activation domains (VP64, p65) $(78,79)$ or with dCas9 bound to modified gRNA scaffolds $(80,81)$, and transcriptional repression with dCas9 fused to the KRAB transcriptional repression domain (CRISPRi) (82). With these, three different branches of CRISPR genome-wide screening methods have been developed: arrayed, phenotypic pooled, and single-cell screens. In arrayed screens, the experimental design revolves around multi-well plate experiments, with wells being exposed to a single sgRNA at a time, a costly and time-consuming process with limited throughput. As such, most recent genome-wide screens have relied on the pooled phenotypic approach, with single-cell CRISPR/Cas9 screens becoming increasingly popular.

\section{Pooled CRISPR screens with CRISPR/Cas9}

In pooled screens, a pool of cells is exposed to a library of sgRNAs, after which phenotypic analysis (selection) is done. Importantly, this sgRNA library is both diverse and redundant. Multiple target sequences (exons or regulatory elements of genes) are selected, and multiple guides for each target are designed to mitigate false-negative results. As an advantage, many pre-defined libraries that have been optimized for various applications are available (83). In very general terms, a pooled genome-wide CRISPR/Cas9 screen involves four key steps (Fig. 3): 1) the generation and packaging of an sgRNA library that targets genes of interest, usually using lentivirus vectors, 2) transduction of the sgRNA library into cell lines of interest that express or contain a variation of Cas9, 3) selection of transduced cells following various challenges (such as infection or drug inhibition), and 4) retrieval of genetic information from enriched and control cells using NGS (84).

Pooled screens can rapidly identify therapeutic targets for a

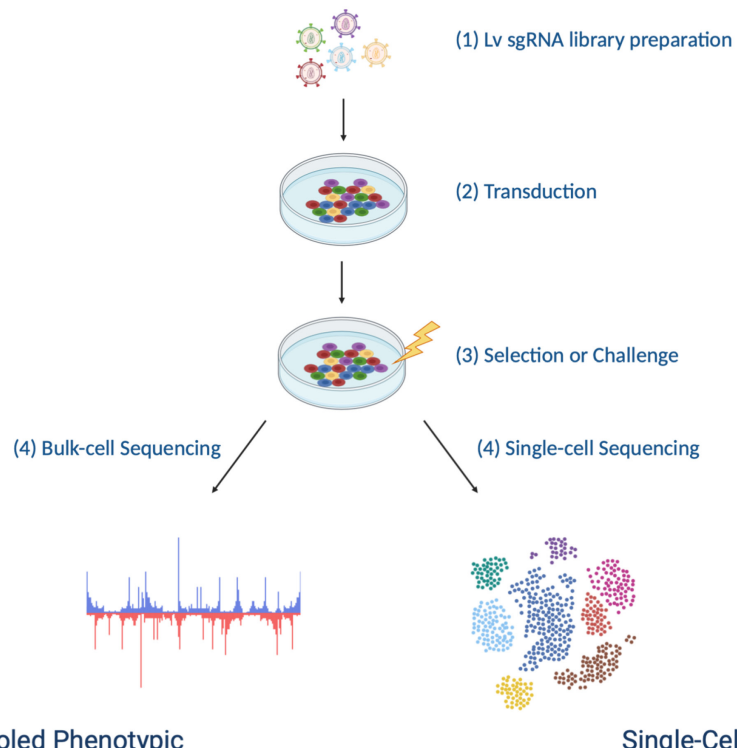

Pooled Phenotypic

Single-Cell

Fig. 3. Pooled phenotypic and single-cell genome-wide screening (1) A lentiviral sgRNA library is constructed based on the genes of interest and (2) transduced into cells. Next, the cells are (3) challenged according to the experimental setup and fed into (4) bulk- or single-cell sequencing workflows.

multitude of diseases, both genetic and infectious. For HIV, a pooled screen was used in CD4 + cells to identify novel factors involved in internalization. The study identified TPST2 and SLC35B2, two members of the CCR5 sulfation pathway that was previously reported to be crucial for the interactions with the gp120 of HIV. Indeed, the independent knock-out of these proteins significantly decreased the rate of infection of the CCR5-tropic JR-CSF HIV strain against a human CD4 + T-cell line and primary $T$ cells (85). Further, since combination antiretroviral therapy alone cannot cure HIV, many groups have tried to find mechanisms involved in HIV latency that can be targeted using latency-reversal agents with the aim of depleting latently-infected cells (86). One study used a pooled screen to identify a set of deubiquitinases involved in the latency of HIV. Among these were UCH37, USP14, OTULIN, and USP5, with USP14 being confirmed pharmacologically (87). With these results, the authors proposed that these enzymes could have a direct role on HIV-1 latency reversal by regulating NFkB signalling, which has been shown with other deubiquitinses (88).

Pooled screens have also been applied to key regulatory genes in immune cells involved in cancer immunity. Pan et al. sought to define key factors in the tumor resistance to CD8 + T cell-mediated killing and thus identify novel potential targets for therapeutics. In the B16F10 melanoma cell line, which is resistant to anti-PD-1/CTLA-4 blockade therapy, a genome-wide pool of 79,637 sgRNAs was used to identify Arid2 and Pbrm1, which, when independently disrupted in cancer cells, increased 
tumor-killing activity by Pmel-1 and OT-I cytotoxic T cells (89). These genes are involved in chromatin remodelling (90) and, when disrupted, are associated with an increased sensitivity to interferon- $\gamma$ and an increased secretion of T-cell recruiting chemokines CXCL9 and CXCL10. These factors collectively led to a favorable tumor microenvironment. As a result, tumor growth was significantly delayed in $\mathrm{Pbrm}^{-/-}$mice with checkpoint-blockade therapy.

Another study attempted to elucidate factors involved in T-cell exhaustion, one of the major mechanisms in the failure of CD-19-specific CAR-T-cell therapy (CAR-T19). They used the Brunello lentiviral sgRNA library targeting nearly 20,000 genes to probe factors that conferred resistance of a CD19+ acute lymphoblastic leukemia (ALL) to CAR-T19 therapy. They found that FADD ${ }^{\mathrm{KO}}$ and BID ${ }^{\mathrm{KO}}$ Nalm-6 cells, a human CD19+ leukemia cell line, were more resistant to CAR-T19 treatment both in vitro and when implanted in immune-deficient $\mathrm{NOD} / \mathrm{SCID} / \gamma^{-1-}$ mice (91). Resistance in this context was associated with the decreased killing of the cancer cells and therefore persistence of the antigen, which led to CAR-T19 exhaustion. These results were consistent with analysis of non-responders of Tisagenlecleucel, which showed significantly decreased expression of death-receptor-associated proapoptotic genes.

Last, another study attempted to identify novel regulators of Foxp3, a master transcriptional regulator in $\mathrm{T}_{\text {reg }}$ cells. By targeting 489 nuclear factors, they identified Usp22, which is part of the deubiquitination module of the SAGA chromatin-modifying complex, as being a positive regulator of Foxp3 (92). Indeed, Usp22 $2^{\text {Treg-KO }}$ B6 mice displayed a delayed increase in EG7 lymphoma volume, which was associated with an increase in CD4 + and CD8 + tumor-infiltrating lymphocytes, likely because there were fewer tumor-infiltrating $\mathrm{T}_{\text {reg }}$ cells. These studies and others support the use of genome-wide pooled phenotypic screens in the elucidation of disease-related mechanisms that might lead to the discovery of novel therapeutics.

\section{Single-cell screens with CRISPR}

Single-cell CRISPR/Cas9 screens are used to study the correlation between small genomic perturbations and global transcriptome changes at a single-cell level for a more comprehensive dataset than can be provided by pooled phenotypic screens (93). Several platforms have recently been developed, each of which relies on massively parallel RNA single-cell sequencing (MARS-seq) or droplet-based barcoding (DROP-Seq) $(94,95)$. For example, DROP-Seq uses microfluidic channels to pair a single cell with a hydrogel-encased bead attached to oligonucleotides containing unique cell barcodes $(\mathrm{CBC})$ and unique molecular identifiers (UMI). The CBC pairs CDNA to a single cell, whereas the UMI is used for quantification. This singlecell-bead pair is then incorporated into a nanolitre-sized droplet containing cell lysis buffer and a RT-PCR enzyme mix for cDNA synthesis and subsequent sequencing. Several single-cell CRISPR/Cas9 platforms using these technologies have been independently developed in parallel and are named Perturb-Seq (96), Mosaic-Seq (97), CRISP-Seq (98), and CROP-Seq (99). Generally, these platforms use a standard sgRNA lentiviral library with a key modification, such as a polyadenylated unique guide index (UGl), to find the sgRNA identity within the transcriptome. The applications of single-cell CRISPR screening has been used extensively in non-immune cells but will not be covered here $(94,100,101)$.

In its development, CRISP-Seq was used to probe immune genetic circuits in myeloid cells and identified key transcription factors involved in lineage commitment and response to pathogens. To do this, the authors transduced Cas9+ bone-marrow cells with a panel of sgRNAs targeting transcription factors involved in immune-cell development and responses, and [1] in vitro cultured or [2] in vivo engrafted the perturbed cells, followed by LPS challenge and single-cell analysis on CD11c+ myeloid cells. With this model, they identified opposing roles for Irf8 and Cebpb (98). Irf8 was involved in the lineage commitment of dendritic cells (DC), whereas Cebpb was essential for monocyte development. Further, they confirmed the known roles for Rela and Stat $1 / 2$ in the inflammatory and antiviral responses in different myeloid-cell subpopulations and found an unexpected correlation between Rela and antiviral responses in dendritic cells. In a similar model, Dixit et al. used Perturb-Seq to distinguish the effects of 24 transcription factors that are involved in cell-lineage commitment and response to pathogens in myeloid cells. They also found that the pioneer factors Cebpb and Hif1 $\alpha$ were involved in inflammatory gene expression (TNF and ROS), whereas PU.1 led to their downregulation (96). The latter result was consistent with the recent finding by Chopin et al. that PU. 1 is involved in conventional dendritic-cell development (102). Next, they confirmed the known roles of Stat $1 / 2$ in the activation of the antiviral response and the roles of Rel, Irf2, and Atf3 in its suppression. An interesting consistency between these methods was the finding that Cebpb, which is involved in inflammatory monocyte development, has roles opposite to those of Irf8 and PU.1, which are involved in dendritic-cell commitment, suggesting significant crosstalk between different pioneering factors that determine cell fate.

\section{CONCLUDING REMARKS}

Developments in CRISPR/Cas9 technology have revolutionized our understanding of disease-related mechanisms and are allowing research for potentially ground-breaking therapies. Despite its advantages, this system should be further optimized to mitigate safety concerns. For example, the generation of DSBs activates p53 (103) and induces megabase-scale chromosomal truncations (104). This can be overcome with precision editing methods, such as base editing and prime editing, that do not rely on DSB. Indeed, it has recently been shown that multiplexed gene knock-out can be accomplished by using base editing (105). Next, although the AAV-based donor delivery is 
highly efficient in HDR-based knock-in, the time and costs for its manufacturing limit its broad applicability, and as such, non-viral DNA delivery is being optimized. As an interesting future direction, since the efficiency of HDR mediated knock-in can be limited by endogenous DNA-repair machinery, the use of self-sufficient integration machinery, such as transposases, could be an alternative method, and has been used in $E$. coli with marginal efficiency and accuracy $(106,107)$. With future improvements, CRISPR/Cas9 will become an indispensable tool for unraveling novel immune pathways and developing therapies for diseases with no curative therapeutic options.

\section{CONFLICTS OF INTEREST}

The authors have no conflicting interests.

\section{REFERENCES}

1. Mardis ER (2017) DNA sequencing technologies: 20062016. Nat Protoc 12, 213-218

2. Zhang W, Mitchell LA, Bader JS and Boeke JD (2020) Synthetic genomes. Annu Rev Biochem 89, 77-101

3. Pickar-Oliver A and Gersbach CA (2019) The next generation of CRISPR-Cas technologies and applications. Nat Rev Mol Cell Biol 20, 490-507

4. Sander JD and Joung JK (2014) CRISPR-Cas systems for editing, regulating and targeting genomes. Nat Biotechnol 32, 347-355

5. Anzalone AV, Koblan LW and Liu DR (2020) Genome editing with CRISPR-Cas nucleases, base editors, transposases and prime editors. Nat Biotechnol 38, 824-844

6. Tong S, Moyo B, Lee CM, Leong K and Bao G (2019) Engineered materials for in vivo delivery of genome-editing machinery. Nat Rev Mater 4, 726-737

7. Ishino $Y$, Shinagawa $H$, Makino $K$, Amemura $M$ and Nakata A (1987) Nucleotide sequence of the iap gene, responsible for alkaline phosphatase isozyme convertsion in Escherichia coli, and identification of the gene product. J Bacteriol 169, 5429-5433

8. Jansen R, Embden JD, Gaastra W and Schouls LM (2002) Identification of genes that are associated with DNA repeats in prokaryotes. Mol Microbiol 43, 1565-1575

9. Mojica FJ, Diez-Villasenor C, Garcia-Martinez J and Soria E (2005) Intervening sequences of regularly spaced prokaryotic repeats derive from foreign genetic elements. J Mol Evol 60, 174-182

10. Barrangou R, Fremaux C, Deveau H et al (2007) CRISPR provides acquired resistance against viruses in prokaryotes. Science 315, 1709-1712

11. Makarova KS, Wolf YI, Iranzo J et al (2020) Evolutionary classification of CRISPR-Cas systems: a burst of class 2 and derived variants. Nat Rev Microbiol 18, 67-83

12. Jinek M, Chylinski K, Fonfara I, Hauer M, Doudna JA and Charpentier E (2012) A programmable dual-RNA-guided DNA endonuclease in adaptive bacterial immunity. Science 337, 816-821

13. Mojica FJM, Diez-Villasenor C, Garcia-Martinez J and Almendros C (2009) Short motif sequences determine the targets of the prokaryotic CRISPR defence system. Microbiology (Reading) 155, 733-740

14. Wang H, La Russa M and Qi LS (2016) CRISPR/Cas9 in genome editing and beyond. Annu Rev Biochem 85, 227-264

15. Ren J and Zhao Y (2017) Advancing chimeric antigen receptor T cell therapy with CRISPR/Cas9. Protein Cell 8, 634-643

16. Kim S, Kim D, Cho SW, Kim J and Kim JS (2014) Highly efficient RNA-guided genome editing in human cells via delivery of purified Cas9 ribonucleoproteins. Genome Res 24, 1012-1019

17. Hendel A, Bak RO, Clark JT et al (2015) Chemically modified guide RNAs enhance CRISPR-Cas genome editing in human primary cells. Nat Biotechnol 33, 985-989

18. Hornung $V$ and Latz E (2010) Intracellular DNA recognition. Nat Rev Immunol 10, 123-130

19. Geering B and Fussenegger M (2015) Synthetic immunology: modulating the human immune system. Trends Biotechnol 33, 65-79

20. Wei SC, Duffy CR and Allison JP (2018) Fundamental mechanisms of immune checkpoint blockade therapy. Cancer Discov 8, 1069-1086

21. Klinger $M$, Benjamin J, Kischel R, Stienen $S$ and Zugmaier G (2016) Harnessing T cells to fight cancer with BiTE(R) antibody constructs--past developments and future directions. Immunol Rev 270, 193-208

22. Guedan S, Ruella $M$ and June $\mathrm{CH}$ (2019) Emerging cellular therapies for cancer. Annu Rev Immunol 37, 145-171

23. Schuster SJ, Svoboda J, Chong EA et al (2017) Chimeric antigen receptor T cells in refractory B-cell lymphomas. N Engl J Med 377, 2545-2554

24. Neelapu SS, Locke FL, Bartlett NL et al (2017) Axicabtagene ciloleucel CAR T-cell therapy in refractory large B-cell lymphoma. N Engl J Med 377, 2531-2544

25. Maude SL, Laetsch TW, Buechner J et al (2018) Tisagenlecleucel in children and young adults with B-cell lymphoblastic leukemia. N Engl J Med 378, 439-448

26. Blank CU, Haining WN, Held W et al (2019) Defining 'T cell exhaustion'. Nat Rev Immunol 19, 665-674

27. Wherry EJ and Kurachi M (2015) Molecular and cellular insights into T cell exhaustion. Nat Rev Immunol 15, 486499

28. Choi BD, Yu X, Castano AP et al (2019) CRISPR-Cas9 disruption of PD-1 enhances activity of universal EGFRvIlI CAR T cells in a preclinical model of human glioblastoma. J Immunother Cancer 7, 304

29. Hu W, Zi Z, Jin Y et al (2019) CRISPR/Cas9-mediated PD-1 disruption enhances human mesothelin-targeted CAR $\mathrm{T}$ cell effector functions. Cancer Immunol Immunother 68, 365-377

30. Rupp LJ, Schumann K, Roybal KT et al (2017) CRISPR/ Cas9-mediated PD-1 disruption enhances anti-tumor efficacy of human chimeric antigen receptor T cells. Sci Rep 7, 737

31. Su S, Hu B, Shao J et al (2016) CRISPR-Cas9 mediated efficient PD-1 disruption on human primary T cells from cancer patients. Sci Rep 6, 20070

32. Stadtmauer EA, Fraietta JA, Davis MM et al (2020) 
CRISPR-engineered T cells in patients with refractory cancer. Science 367, eaba7365

33. Yang L, Pang Y and Moses HL (2010) TGF-beta and immune cells: an important regulatory axis in the tumor microenvironment and progression. Trends Immunol 31, 220-227

34. Tang N, Cheng C, Zhang X et al (2020) TGF-beta inhibition via CRISPR promotes the long-term efficacy of CAR T cells against solid tumors. JCI Insight 5, e133977

35. Yamada A, Arakaki R, Saito $M$, Kudo $Y$ and Ishimaru $N$ (2017) Dual role of Fas/FasL-mediated signal in peripheral immune tolerance. Front Immunol 8, 403

36. Ren J, Zhang X, Liu X et al (2017) A versatile system for rapid multiplex genome-edited CAR $T$ cell generation. Oncotarget 8, 17002-17011

37. Riese MJ, Moon EK, Johnson BD and Albelda SM (2016) Diacylglycerol kinases (DGKs): novel targets for improving $T$ cell activity in cancer. Front Cell Dev Biol 4, 108

38. Jung IY, Kim YY, Yu HS, Lee M, Kim S and Lee J (2018) CRISPR/Cas9-mediated knockout of DGK improves antitumor activities of human T cells. Cancer Res 78, 46924703

39. Norelli M, Camisa B, Barbiera G et al (2018) Monocytederived IL-1 and IL-6 are differentially required for cytokine-release syndrome and neurotoxicity due to CAR T cells. Nat Med 24, 739-748

40. Teachey DT, Lacey SF, Shaw PA et al (2016) Identification of predictive biomarkers for cytokine release syndrome after chimeric antigen receptor T-cell therapy for acute lymphoblastic leukemia. Cancer Discov 6, 664-679

41. Sterner RM, Sakemura R, Cox MJ et al (2019) GM-CSF inhibition reduces cytokine release syndrome and neuroinflammation but enhances CAR-T cell function in xenografts. Blood 133, 697-709

42. Alcantara $\mathrm{M}$, Tesio $\mathrm{M}$, June $\mathrm{CH}$ and Houot $\mathrm{R}$ (2018) CAR T-cells for T-cell malignancies: challenges in distinguishing between therapeutic, normal, and neoplastic T-cells. Leukemia 32, 2307-2315

43. Gomes-Silva D, Srinivasan M, Sharma S et al (2017) CD7-edited T cells expressing a CD7-specific CAR for the therapy of T-cell malignancies. Blood 130, 285-296

44. Ren J, Liu X, Fang C, Jiang S, June $\mathrm{CH}$ and Zhao $\mathrm{Y}$ (2017) Multiplex genome editing to generate universal CAR T cells resistant to PD1 inhibition. Clin Cancer Res 23, 2255-2266

45. Morton LT, Reijmers RM, Wouters AK et al (2020) Simultaneous deletion of endogenous TCRalphabeta for TCR gene therapy creates an improved and safe cellular therapeutic. Mol Ther 28, 64-74

46. Maher J, Brentjens RJ, Gunset G, Riviere I and Sadelain M (2002) Human T-lymphocyte cytotoxicity and proliferation directed by a single chimeric TCRzeta /CD28 receptor. Nat Biotechnol 20, 70-75

47. Milone MC, Fish JD, Carpenito C et al (2009) Chimeric receptors containing CD137 signal transduction domains mediate enhanced survival of $\mathrm{T}$ cells and increased antileukemic efficacy in vivo. Mol Ther 17, 1453-1464

48. Uren AG, Kool J, Berns A and van Lohuizen M (2005) Retroviral insertional mutagenesis: past, present and future. Oncogene 24, 7656-7672

49. Niederer HA and Bangham CR (2014) Integration site and clonal expansion in human chronic retroviral infection and gene therapy. Viruses 6, 4140-4164

50. Woods NB, Muessig A, Schmidt M et al (2003) Lentiviral vector transduction of NOD/SCID repopulating cells results in multiple vector integrations per transduced cell: risk of insertional mutagenesis. Blood 101, 1284-1289

51. Eyquem J, Mansilla-Soto J, Giavridis T et al (2017) Targeting a CAR to the TRAC locus with CRISPR/Cas9 enhances tumour rejection. Nature 543, 113-117

52. Wright JF (2008) Manufacturing and characterizing AAVbased vectors for use in clinical studies. Gene Ther 15, 840-848

53. Roth TL, Puig-Saus C, Yu R et al (2018) Reprogramming human $T$ cell function and specificity with non-viral genome targeting. Nature 559, 405-409

54. Nguyen DN, Roth TL, Li PJ et al (2020) Polymer-stabilized Cas9 nanoparticles and modified repair templates increase genome editing efficiency. Nat Biotechnol 38, 44-49

55. Roth TL, Li PJ, Blaeschke F et al (2020) Pooled Knockin Targeting for Genome Engineering of Cellular Immunotherapies. Cell 181, 728-744 e721

56. Tothova Z, Krill-Burger JM, Popova KD et al (2017) Multiplex CRISPR/Cas9-based genome editing in human hematopoietic stem cells models clonal hematopoiesis and myeloid neoplasia. Cell Stem Cell 21, 547-555.e8

57. Wagenblast E, Azkanaz M, Smith SA et al (2019) Functional profiling of single CRISPR/Cas9-edited human long-term hematopoietic stem cells. Nat Commun 10, 4730

58. Cavazzana M, Antoniani C and Miccio A (2017) Gene therapy for beta-hemoglobinopathies. Mol Ther 25, 11421154

59. Dever DP, Bak RO, Reinisch A et al (2016) CRISPR/Cas9 beta-globin gene targeting in human haematopoietic stem cells. Nature 539, 384-389

60. Wu Y, Zeng J, Roscoe BP et al (2019) Highly efficient therapeutic gene editing of human hematopoietic stem cells. Nat Med 25, 776-783

61. Zeng J, Wu Y, Ren C et al (2020) Therapeutic base editing of human hematopoietic stem cells. Nat Med 26, 535-541

62. Traxler EA, Yao Y, Wang YD et al (2016) A genome-editing strategy to treat beta-hemoglobinopathies that recapitulates a mutation associated with a benign genetic condition. Nat Med 22, 987-990

63. Weber L, Frati G, Felix T et al (2020) Editing a gammaglobin repressor binding site restores fetal hemoglobin synthesis and corrects the sickle cell disease phenotype. Sci Adv 6, eaay9392

64. Hacein-Bey-Abina S, Garrigue A, Wang GP et al (2008) Insertional oncogenesis in 4 patients after retrovirus-mediated gene therapy of SCID-X1. J Clin Invest 118, 31323142

65. Woods NB, Bottero V, Schmidt $M$, von Kalle $C$ and Verma IM (2006) Gene therapy: therapeutic gene causing lymphoma. Nature 440, 1123

66. Pavel-Dinu M, Wiebking V, Dejene BT et al (2019) Gene correction for SCID-X1 in long-term hematopoietic stem cells. Nat Commun 10, 1634

67. Holt N, Wang J, Kim K et al (2010) Human hemato- 
poietic stem/progenitor cells modified by zinc-finger nucleases targeted to CCR5 control HIV-1 in vivo. Nat Biotechnol 28, 839-847

68. Shi B, Li J, Shi X et al (2017) TALEN-mediated knockout of CCR5 confers protection against infection of human immunodeficiency virus. J Acquir Immune Defic Syndr 74, 229-241

69. Xu L, Yang H, Gao Y et al (2017) CRISPR/Cas9-mediated CCR5 ablation in human hematopoietic stem/progenitor cells confers HIV-1 resistance in vivo. Mol Ther 25, 17821789

70. Xu L, Wang J, Liu Y et al (2019) CRISPR-edited stem cells in a patient with HIV and acute lymphocytic leukemia. N Engl J Med 381, 1240-1247

71. Hartweger H, McGuire AT, Horning M et al (2019) HIVspecific humoral immune responses by CRISPR/Cas9edited B cells. J Exp Med 216, 1301-1310

72. Moffett HF, Harms CK, Fitzpatrick KS, Tooley MR, Boonyaratanakornkit J and Taylor JJ (2019) B cells engineered to express pathogen-specific antibodies protect against infection. Sci Immunol 4, eaax0644

73. McManus MT and Sharp PA (2002) Gene silencing in mammals by small interfering RNAs. Nat Rev Genet 3, 737-747

74. Jackson AL and Linsley PS (2010) Recognizing and avoiding siRNA off-target effects for target identification and therapeutic application. Nat Rev Drug Discov 9, 57-67

75. Munoz DM, Cassiani PJ, Li L et al (2016) CRISPR screens provide a comprehensive assessment of cancer vulnerabilities but generate false-positive hits for highly amplified genomic regions. Cancer Discov 6, 900-913

76. Tian X, Gu T, Patel S, Bode AM, Lee MH and Dong Z (2019) CRISPR/Cas9 - an evolving biological tool kit for cancer biology and oncology. NPJ Precis Oncol 3, 8

77. Wang T, Wei JJ, Sabatini DM and Lander ES (2014) Genetic screens in human cells using the CRISPR-Cas9 system. Science 343, 80-84

78. Mali P, Aach J, Stranges PB et al (2013) CAS9 transcriptional activators for target specificity screening and paired nickases for cooperative genome engineering. Nat Biotechnol 31, 833-838

79. Perez-Pinera P, Kocak DD, Vockley CM et al (2013) RNA-guided gene activation by CRISPR-Cas9-based transcription factors. Nat Methods 10, 973-976

80. Konermann S, Brigham MD, Trevino AE et al (2015) Genome-scale transcriptional activation by an engineered CRISPR-Cas9 complex. Nature 517, 583-588

81. Zalatan JG, Lee ME, Almeida R et al (2015) Engineering complex synthetic transcriptional programs with CRISPR RNA scaffolds. Cell 160, 339-350

82. Gilbert LA, Larson MH, Morsut L et al (2013) CRISPR-mediated modular RNA-guided regulation of transcription in eukaryotes. Cell 154, 442-451

83. McDade JR, Waxmonsky NC, Swanson LE and Fan M (2016) Practical considerations for using pooled lentiviral CRISPR libraries. Curr Protoc Mol Biol 115, 31.5.131.5.13

84. Joung J, Konermann S, Gootenberg JS et al (2017) Genome-scale CRISPR-Cas9 knockout and transcriptional activation screening. Nat Protoc 12, 828-863
85. Park RJ, Wang T, Koundakjian D et al (2017) A genomewide CRISPR screen identifies a restricted set of HIV host dependency factors. Nat Genet 49, 193-203

86. Ait-Ammar A, Kula A, Darcis G et al (2019) Current status of latency reversing agents facing the heterogeneity of HIV-1 cellular and tissue reservoirs. Front Microbiol 10, 3060

87. Rathore A, Iketani S, Wang $\mathrm{P}$, Jia M, Sahi V and Ho DD (2020) CRISPR-based gene knockout screens reveal deubiquitinases involved in HIV-1 latency in two Jurkat cell models. Sci Rep 10, 5350

88. Manganaro L, Pache L, Herrmann T et al (2014) Tumor suppressor cylindromatosis (CYLD) controls HIV transcription in an NF-kappaB-dependent manner. J Virol 88, 75287540

89. Pan D, Kobayashi A, Jiang P et al (2018) A major chromatin regulator determines resistance of tumor cells to $T$ cell-mediated killing. Science 359, 770-775

90. Kadoch C and Crabtree GR (2015) Mammalian SWI/SNF chromatin remodeling complexes and cancer: Mechanistic insights gained from human genomics. Sci Adv 1, e1500447

91. Singh N, Lee YG, Shestova O et al (2020) Impaired death receptor signaling in leukemia causes antigen- independent resistance by inducing CAR T-cell dysfunction. Cancer Discov 10, 552-567

92. Cortez JT, Montauti E, Shifrut E et al (2020) CRISPR screen in regulatory $\mathrm{T}$ cells reveals modulators of Foxp3. Nature 582, 416-420

93. Henriksson J (2019) CRISPR screening in single cells. Methods Mol Biol 1979, 395-406

94. Klein AM, Mazutis L, Akartuna I et al (2015) Droplet barcoding for single-cell transcriptomics applied to embryonic stem cells. Cell 161, 1187-1201

95. Macosko EZ, Basu A, Satija R et al (2015) Highly parallel genome-wide expression profiling of individual cells using nanoliter droplets. Cell 161, 1202-1214

96. Dixit A, Parnas O, Li B et al (2016) Perturb-Seq: dissecting molecular circuits with scalable single-cell RNA profiling of pooled genetic screens. Cell 167, 1853-1866. e17

97. Xie S, Duan J, Li B, Zhou P and Hon GC (2017) Multiplexed engineering and analysis of combinatorial enhancer activity in single cells. Mol Cell 66, 285-299.e5

98. Jaitin DA, Weiner A, Yofe I et al (2016) Dissecting immune circuits by linking CRISPR-pooled screens with single-cell RNA-Seq. Cell 167, 1883-1896.e15

99. Datlinger P, Rendeiro AF, Schmidl C et al (2017) Pooled CRISPR screening with single-cell transcriptome readout. Nat Methods 14, 297-301

100. Adamson B, Norman TM, Jost M et al (2016) A multiplexed single-cell CRISPR screening platform enables systematic dissection of the unfolded protein response. Cell 167, 1867-1882.e21

101. McFaline-Figueroa JL, Hill AJ, Qiu X, Jackson D, Shendure J and Trapnell C (2019) A pooled single-cell genetic screen identifies regulatory checkpoints in the continuum of the epithelial-to-mesenchymal transition. Nat Genet 51, 1389-1398

102. Chopin M, Lun AT, Zhan $Y$ et al (2019) Transcription 
factor PU.1 promotes conventional dendritic cell identity and function via induction of transcriptional regulator DC-SCRIPT. Immunity 50, 77-90.e5

103. Haapaniemi E, Botla S, Persson J, Schmierer B and Taipale J (2018) CRISPR-Cas9 genome editing induces a p53-mediated DNA damage response. Nat Med 24, 927930

104. Cullot G, Boutin J, Toutain J et al (2019) CRISPR-Cas9 genome editing induces megabase-scale chromosomal truncations. Nat Commun 10, 1136
105. Webber BR, Lonetree CL, Kluesner MG et al (2019) Highly efficient multiplex human $\mathrm{T}$ cell engineering without double-strand breaks using Cas9 base editors. Nature Communications 10,5222

106. Strecker J, Ladha A, Gardner Z et al (2019) RNA-guided DNA insertion with CRISPR-associated transposases. Science 365, 48-53

107. Klompe SE, Vo PLH, Halpin-Healy TS and Sternberg SH (2019) Transposon-encoded CRISPR-Cas systems direct RNA-guided DNA integration. Nature 571, 219-225 